are physiologic and psychologic facts within the observation of any one. They are also unknown pathologic conditions, of both defective growth, and exhaustion and poisoning. The normal or approximately healthy man is not predisposed to use alcohol or narcotic drugs. While alcohol is not pleasing alone, when mixed in water and flavoring substances it may be tolerated, but when its effects are realized a natural intolerance begins. Unlike any other drug it conceals the conditions which it provokes, until such a time when they become fixed, then repugnance begins. This persistent concealment breaks out in the next generation as a physiologic bias and tendency to use the same drugs, to conceal the degeneration produced by the same causes. This persistent poisoning incapacitates and limits the vigor of the next life, giving it certain impulses to exhaustion; and brealing up its power of adaptability, causes pain and suffering which seeks relief in the same way. These subtle forces of heredity and morbid impulses, whose causes are only faintly comprehended, are expressed in these terms, intolerance and predisposition. Along this line we need new studies.

\section{ALCOHOL IN THE GRIPPE.}

Read in the Section on State Medicine at the Forty-sixth Anuual Meeting of the American Medical Association, at Baltimore, Ard., May $7-10,1895$.

BY CHARLES H. SHEPARD, M.D. RROOKLYN, N. Y.

The present form of the grippe is an infectious disease finding its way through the respiratory mucous membrane, and the resulting toxins, by their migrations, tend to induce a lasting lassitude and depression that are characteristic symptoms in many of the cases. The primary cause of this disease is the susceptibility of the system. This is shown by the fact that some persons enjoy entire freedom, while others succumb to its influence every time it appears.

There are many conditions that favor it. What is called a poor circulation is accompanied by an inability to throw off morbific influences, and persons in that condition are likely to suffer from every epidemic. The same applies to indigestion, for when the body is not well nourished there is but feeble reactive force. Those having a torpid liver find the grippe an added burden to the discomfort of life, and those with an inactive skin are still more likely to suffer from its baneful influence, but among the more prominent causes of susceptibility may be classed the almost universal habit of drug taking, from the mild tonics, to patent medicines. Whenever one is out of order in any way, the first thing seems to be to swallow something, instead of trying to find out the cause of the trouble and seeking to obviate it by reguIating the habits of life. This custom tends continually to lower the tone of the system, and the more it is indulged in the more evident becomes the apparent necessity of continuing the down-hill course. A large share of the deterioriation of our people is due to the use of alcoholic tonics, or stimulants, as they are sometimes called. The majority do not look beyond the fact that they seem to feel better after their use, but this feeling comes from the benumbing action of the alcohol itself, and never from any building up action of the drug, because it has no such action. What is supposed by some to be that action is only an irritation resulting from the efforts of the system to get rid of the drug. It only exhausts the vitality so much quicker, and therefore alcohol should always be considered a poison to the human system, and is already so considered by some of the highest medical authorities. The cells of the brain lie buried in a nutrient plasma, and their vigor depends upon the quality of this plasma. Should this become impregnated with alcohol in even a small degree, the action would necessarily be, so far, an abnormal one. These cells become exhausted from their natural activity and are then shrunken, depending for renewal on rest and the nutrient plasma by which they are surrounded. This has been demonstrated by experiments on dogs and pigeons. Out of a number, some were killed, and on examination the brain cells were found well nourished and full, while their companions, after exercise and fatigue, showed shrunken brain cells.

Irritability always follows exhaustion in a nerve cell, by whatever cause excited, and there is every reason to believe that insomnia, neurasthenia, and acute insanity are due to fatigue of the nerve cells through failure of the nutrient plasma to furnish the necessary nutriment for restoration. There is a collapse that frequently comes after the use of so-called tonics and stimulants. Outraged nature cries aloud for help. This it is that causes so many to return to their cups. The condition may be called brain and nerve exhaustion. It is found most difficult to treat by ordinary methods, because these nearly all depend upon rousing up the vitality by recourse to some new irritant or drug.

The strain upon the nervous energy of the individual in the pressure of modern civilization is so great that many, not knowing or realizing the inevitable result, and feeling that there is no time for rest and recuperation, seek in alcohol or some narcotic a surcease for their jaded feelings. This, with some more quickly than with others, is sure to bring about a condition of neurasthenia, so aptly termed by Dr. Beard a chronic functional disease of the nervous system, the basis of which is impoverishment of the nervous tissue in excess of repair, which may exist without any evidence of profound organic complication. We have daily before our eyes a profusion of examples of the various forms of nervous prostration. Experts can readily recognize in their patients underlying symptoms of nerve exhaustion, which their personal record showed had long antedated the expression of any distinct climax of disease.

It is difficult for the ordinary mind to see the end from the beginning, for while some individuals, probably from inheritance from a sound ancestry, are enabled to ignore the laws of health for a long time, the superficial observer can point to them with pride and say: "They do no harm, in moderation." Even the Royal Commission of England reports that the temperate use of opium in India should be viewed in the same light as the temperate use of alcohol in England, which according to their measure is harmful or harmless according to the amount and discretion with which it is used. This is altogether too much of a commercial report. It needs a commis. sion from America, France, or possibly Germany, to get a perfectly unbiased opinion. The question of the toleration of narcotics is not settled and never will be until it is settled right. The consensus of medical opinion is coming more and more to the conclusion that alcohol is only and always a poison, and 
therefore has no place in the human economy. Such the isolation of cases tends to restrict the spread of authority as Dr. Forel, the famous specialist of the the disease, but when the best conditions are secured University of Zurich, says: "Alcohol, even when such a course is unnecessary.

diluted as in wine, beer and cider, is a poison which changes pathologically the tissues of the body and leads to fatty degeneration. I am one who assigns but a very subordinate position to alcohol as a medicine."

The value of rest and passive exercise is receiving universal recognition as a means of forestalling the cause of disease and building up physiologic barriers against its invasion. Brain workers should secure at least one month's vacation out of the twelve. There

For these reasons we contend that alcohol has no is no new order of sleep, of rest, or of nutrition. place in the treatment of grippe; on the contrary, it Chemistry can never supply this need, and as is because of the too frequent use of this and other all nerve derangement is indicative of innutrition, narcotics that epidemics, particularly the grippe, the first step toward recovery is rest of mind and make such fearful headway in our land, and such body.

must inevitably be the rule until our people study the laws of health and obey them.

The injurious and deteriorating effect of the use of any narcotic, tobacco not excepted, is daily demonstrated to those who have eyes to see. Too frequently there is to be found in the daily papers an account of the physical wreck of some young man from the excessive use of the drug. As the future of our nation depends upon the young, too much can not be done to save them from physical degeneracy. Tea and coffee also belong in the same category of irritant drugs, which by rousing the vital forces to get rid of the poison, provoke the action which is mistakenly supposed to be an added force, whereas that action is oniy one of self-preservation. It will readily be seen how much supporting or building-up of the system there is in such substances by trying to live on them to the exclusion of other things. The result would quickly prove the absurdity of the idea.

Having demonstrated that alcohol is in no sense a relief or remedy for the grippe, it may be well to consider briefly what remedies are offered for this disease, and the reasons why they are used, and also, what is more desirable, present a sure method of prevention and help. For instance, quinin is ranked as a powerful germ destroyer, and its use is considered by many as of paramount importance. Quinin is essentially a nerve poison and capable of producing a profound disturbance of the nervous centers. Practicing physicians are familiar with instances that are rightly accused by patients as having permanently damaged one or another of the nerve functions. A drug of such potency for evil ought to be employed with the greatest care, and never when a milder agency will equally well or better secure the result. Its tolerance by a patient is simply a test of the power of resistance of his nervous system. The increased nervousness of some portions of our population is undoubtedly due to a previous saturating of the system with this recognized anti-periodic. Exceedingly pernicious is the practice that has obtained in some sections of dosing children with this drug. It is sure to lay the foundation of a miserable neurasthenic condition in after life. Attention to the digestive tract by following hygienic and dietetic treatment is the all-important and all-sufficient course to pursue in such cases. Strychnin has been used to some extent. The drug antipyrin is also used, and whenever there is a fever that remedy tends to reduce it, and thus seems to have a good effect, butits action may be more dangerous than the grippe itself. The value of such supposed remedies depends entirely upon the vital reaction of the patient. Again, it is recommended as a sine qua non that the patient should oranges in large quantities, not of two or three, but be confined to the house or bed, with absolute rest, of dozens. The first unpleasant symptoms disappear, according to the severity of the attack. Certainly, and the acid citrate of potash of the juice, by a sim- 
ple chemic action, decreases the amount of fibrin in the blood to an extent which prevents the development of pneumonia."

In our opinion, therefore, the wise $\operatorname{man}$ is he who, with a cheerful spirit, a well-nourished and properly clothed body, uniformly seeks the purifying and invigorating influence of the Turkish bath, and when any such epidemic appears he needs only a little more fervency in his devotions to the religion of cleanliness. It is an important work for us to educate the people to see and understand the far-reaching effectiveness of the Turkish bath, and when large public Turkish baths are demanded by the community, and built by the city, and the people shall have learned to live more in accordance with the laws of health, our land will be freed, not only from the grippe, but from all other forms of pestilence.

Experience proves that the effect of alcohol on the tissues of the body is in every way deteriorating, while experience also proves in the most convincing manner, that the action of the Turkish bath continually tends to renew and build up every tissue. We know also that alcohol interferes with the nutritive processes and hinders elimination, while the Turkish bath promotes the natural action of the nutritive powers, and by throwing off the débris, or used-up tissue, from the system, creates a demand and leaves room for a new supply. Again, it is a fact that alcohol benumbs the nerves and interferes with the coördinating powere of the brain itself, the more alcohol the more disturbance is produced, while the action of the Turkish bath soothes irritated nerves and gives clearer and more powerful mental vision, because it renders more pure the blood that furnishes nutriment to the brain.

It would seem evident to the average intelligence that the Turkish bath is a far more rational remedy for the grippe than drugs or alcohol.

81 Columbia Heights.

\section{FATALITY OF SURGICAL OPERATIONS IN ALCOHOLISM.}

Read in the Section on State Medicine, at the Forty-sixth Annual Meeting of the American Medical Association, at Faltimore, Md., May 7-10. 1895.

BY I. N. QUIMBY, M.D.

JERSEY CITY, N. J.

I thought it might be of some interest to call the attention of this Section of our Association to a form of rheumatism which has frequently come under my observation during the last twenty years, and which has not been fully, I think, recognized as a distinctive form of that disease by the profession. I have had some twenty cases of this form of rheumatism during the above mentioned period.

Not to take up the time of the Section, I will only describe two or three typical cases to illustrate the few remarks I have to make. This form of rheumatism apparently arises wholly from the moderate but continuous use of fermented and distilled liquors and has been aptly termed alcoholic rheumatism.

I think too many of us in our daily rounds in the treatment of the more common forms of diseases, are too apt, for want of time or patient investigation, to fall in the habit of a sort of routine way of diagnosis and treatment, the result of which is often unfortunate to patient and physician. The first case I will describe is somewhat unique.
In the fall of 1875 , I was called to see Mr. M., a builder by profession, aged 48 , who gave me the following history :

He had always been healthy and apparently well, but had been for the previous fifteen years a moderate but continuous user of fermented and distilled liquors, when he was taken with what was described as an acute attack of rheumatism in his left shoulder and elbow joint, with some hoarseness. After several weeks of treatment with colchicum, iodid of potassium, etc., he recovered from the attack of rheumatism, but the hoarseness, which was considerable. still continued to a greater or less degree. He remained in apparent good bealth for about six or seven months when he was again similarly attacked with rheumatism but with increased amount of boarseness, articulating with difficulty. The patient was treated again in about the same way by the same physician, but was relieved less promptly and was left with increased amount of hoarseness. On the third attack, which occurred three or four months after the second attack, I was called to the case, not so much on account of the rheumatism, but on account of the severe attack of aphonia.

No doubt but what there are cases of laryngeal rheumatism occasionally occurring, owing to its complex anatomic structure, and its location between the pharynx and the bronchi, both of which are susceptible of inflammation, separately or in connection with an attack of articular rheumatism, from which he had been suffering for over a year, not being able to speak above a whisper. The laryngeal trouble apparently was the most prominent feature of the present attack.

The patient had been treated by a competent and experienced physician using the most approved remedies for rheumatism and its concomitants at that time.

I felt that there was some complication in the disease or deficiency in treatment, which caused a frequent return of the rheumatism, and this continuous and persistent aphonia; therefore, after a thorough examination of the case and analysis of the urine, and satisfying myself that there was no tumor pressing on the trachea or larynx, and finding that he had continued his moderate yet continuous methods of drinking alcoholic liquors, I arrived at the conclusion that it was this imbibation of alcoholic narcotic irritants which was producing some pathogenic microorganisms in the various tissues of the body, which had much to do, perhaps, with keeping up the rheumatic disturbances and the serious and persistent aphonia from which he has been suffering for over a year, not being able to speak above a whisper, and which was the most prominent feature of the present attack.

I, therefore, began a reconstructive course of treatment, not by giving the general rheumatic remedies, but those of a general tendency to restore the system to its normal condition. I directed that all alcoholic liquors of whatever nature should be immediately discontinued. This was somewhat reluctantly, but immediately complied with. Then I gave the patient 5 gr. calomel, $10 \mathrm{gr}$. bis. sub. carb. $\frac{1}{2} \mathrm{~g}_{2}$. ipecac, followed in a few hours by a saline solution of Rochelle salts. He was also given a saline bath, temperature 90 , for ten minutes, after which he was put upon the following treatment: 